\title{
Froissart bound on total cross section without unknown constants
}

\author{
André Martin ${ }^{1, *}$ and S. M. Roy ${ }^{2, \dagger}$ \\ ${ }^{1}$ Theoretical Physics Division, CERN, 1211 Geneva 23, Switzerland \\ ${ }^{2}$ HBCSE, Tata Institute of Fundamental Research, VN Purav Marg, Mumbai 400088, India
}

(Received 24 June 2013; published 25 February 2014)

\begin{abstract}
We determine the scale of the logarithm in the Froissart bound on total cross sections using absolute bounds on the $D$-wave below threshold for pion-pion scattering. For example, for $\pi^{0} \pi^{0}$ scattering, we show that for c.m. energy $\sqrt{s} \rightarrow \infty, \bar{\sigma}_{\text {tot }}(s, \infty) \equiv s \int_{s}^{\infty} d s^{\prime} \sigma_{\text {tot }}\left(s^{\prime}\right) / s^{\prime 2} \leq \pi\left(m_{\pi}\right)^{-2}\left[\ln \left(s / s_{0}\right)+\right.$ $\left.(1 / 2) \ln \ln \left(s / s_{0}\right)+1\right]^{2}$, where $1 / s_{0}=17 \pi \sqrt{\pi / 2} m_{\pi}^{-2}$.
\end{abstract}

DOI: $10.1103 /$ PhysRevD.89.045015

PACS numbers: 03.67.-a, 03.65.Ud, 42.50.-p

\section{INTRODUCTION}

Froissart [1] proved from the Mandelstam representation that the total cross section $\sigma_{\text {tot }}(s)$ for two particles to go to anything at c.m. energy $\sqrt{s}$ must obey the bound,

$$
\sigma_{\text {tot }}(s) \leq_{s \rightarrow \infty} C\left[\ln \left(s / s_{0}\right)\right]^{2},
$$

where $C, s_{0}$ are unknown constants. Later Martin [2] proved this bound rigorously from axiomatic field theory by enlarging the Lehmann ellipse of analyticity [3] for the absorptive part; further, the constant $C$ was fixed by Lukaszuk and Martin [4] using unitarity and validity of dispersion relations with a finite number of subtractions for $-T<t \leq 0$ (and as a consequence, twice subtracted fixed- $t$ dispersion relations for $\left.|t|<t_{0}[5]\right)$, to obtain

$$
\sigma_{\text {tot }}(s) \leq_{s \rightarrow \infty} 4 \pi /\left(t_{0}-\epsilon\right)\left[\ln \left(s / s_{0}\right)\right]^{2} \equiv \sigma_{\max }(s),
$$

where $t_{0}$ is the lowest singularity in the $t$ channel and $\epsilon$ an arbitrarily small positive constant. For many processes (for example, for $\pi \pi, K K, K \bar{K}, \pi K, \pi N, \pi \Lambda$ scattering), it is known [6] that $t_{0}=4 m_{\pi}^{2}, m_{\pi}$ being the pion mass. (We shall choose units $m_{\pi}=1$.)

These results were obtained by Martin [2] in the framework of local field theory as applied to hadrons, using implicitly the Wightman axioms [7]. However, later, the needed analyticity properties, as well as polynomial boundedness at fixed momentum transfer, were obtained by Epstein et al. [8] in the more general framework of the theory of local observables of Haag et al. [9].

Recently, Azimov has revisited the Froissart bound in a paper [10], Sec. 2 of which is similar to the 1962 and 1963 works of Martin [11]. These papers were a precursor to Martin's later paper [2] which proved the bound rigorously from axiomatic field theory. Azimov has raised doubts about "application of the ideas and methods of axiomatic local field theory to hadron properties." His main point is

\footnotetext{
martina@mail.cern.ch

†smroy@hbcse.tifr.res.in
}

that "hadrons, consisting of quarks and gluons, cannot be pointlike," and might not be associated to local fields. However, Zimmermann [12] has shown that local fields can be associated to composite particles (for instance, deuterons). We postulate that this construction applies to hadrons made of quarks. This is not obvious because, in spite of the practical successes of QCD, nobody knows how to incorporate particles without asymptotic fields in a field theory. Anyway, this is a much weaker assumption than that of the validity of Mandelstam's representation. In particular, we do not use the Froissart-Gribov representation of physical region partial waves for fixed $s$.

The Froissart-Martin bound has triggered much work on high energy theorems (see, e.g., $[13,14]$ ) and on models of high energy scattering [15]. Recently, Martin proved a bound on the total inelastic cross section at high energy [16] which is one fourth of the bound $\sigma_{\max }(s)$ on the total cross section. Wu et al. [17] obtained a bound on $\sigma_{\text {inel }}(s)$ in terms of $\sigma_{\text {tot }}(s)$ which vanishes both when the total cross section vanishes and when it equals the unitarity upper bound.

In spite of all this progress, these bounds share severe shortcomings [17]. (i) They are deduced assuming that the absorptive part $A(s, t), 0 \leq t<t_{0}$ is bounded by Const $s^{2} / \ln \left(s / s_{0}\right)$ for $s \rightarrow \infty$. In fact, the Jin-Martin theorem on twice subtracted dispersion relations only guarantees that

$$
C(t) \equiv \int_{s_{\mathrm{th}}}^{\infty} d s A(s, t) / s^{3}<\infty, \quad 0 \leq t<t_{0},
$$

where $s_{\text {th }}$ is the $s$-channel threshold. As stressed by Yndurain and Common [18], this does not imply that $A(s, t) \leq$ Const. $s^{2} / \ln \left(s / s_{0}\right)$ for all sequences of $s \rightarrow \infty$. (ii) The bounds are expressed in terms of $\sigma_{\max }(s)$, which still contains the unknown scale $s_{0}$ of the logarithm, and the unknown positive parameter $\epsilon$, which can be chosen arbitrarily small but $\neq 0$. If $\epsilon$ is not fixed, $s_{0}$ cannot be fixed, since the advantage of a larger $s_{0}$ can be offset by a larger $\epsilon$. 
We now remove both of these shortcomings. We report definitive bounds on energy averages of the total cross section in which the scale $s_{0}$ is determined in terms of $C(t)$, which is a low energy (in fact below threshold) property in the $t$ channel. In some cases (e.g., for pion-pion scattering), for $t \rightarrow 4, C(t)$ is proportional to the $D$-wave scattering length [19] which is known phenomenologically; hence, we obtain bounds on energy averages in terms of that scattering length. Even more exciting is the fact that for $\pi^{0} \pi^{0}$ scattering we are able to obtain absolute bounds (in terms of pion mass alone) on $C(t)$ below threshold without assuming finiteness of the $D$-wave scattering length; this yields absolute bounds on the asymptotic energy averages of the total cross section.

\section{NORMALIZATIONS}

Let $F(s, t)$ be an $a b \rightarrow a b$ scattering amplitude at c.m. energy $\sqrt{s}$ and momentum transfer squared $t$ normalized for nonidentical particles $a, b$ such that the differential cross section is given by

$$
\frac{d \sigma}{d \Omega}(s, t)=\left|4 \frac{F(s, t)}{\sqrt{s}}\right|^{2},
$$

with $t$ being given in terms of the c.m. momentum $k$ and the scattering angle $\theta$ by the relation,

$$
t=-2 k^{2}(1-\cos \theta) ; \quad z \equiv \cos \theta=1+t /\left(2 k^{2}\right) .
$$

Then, for fixed $s$ larger than the physical $s$-channel threshold, $F(s ; \cos \theta) \equiv F(s, t)$ is analytic in the complex $\cos \theta$ plane inside the Lehmann-Martin ellipse with foci -1 and +1 and semimajor axis $\cos \theta_{0}=1+t_{0} /\left(2 k^{2}\right)$. Within the ellipse, in particular, for $|t|<t_{0}, F(s, t)$ and the $s$-channel absorptive part $F_{s}(s, t)=A(s, t)$ have the convergent partial wave expansions,

$$
\begin{gathered}
F(s, t)=\frac{\sqrt{s}}{4 k} \sum_{l=0}^{\infty}(2 l+1) P_{l}(z) a_{l}(s), \\
F_{s}(s, t)=A(s, t)=\frac{\sqrt{s}}{4 k} \sum_{l=0}^{\infty}(2 l+1) P_{l}(z) \operatorname{Ima}_{l}(s),
\end{gathered}
$$

with the unitarity constraint,

$$
\operatorname{Ima}_{l}(s) \geq\left|a_{l}(s)\right|^{2}, \quad s \geq 4 .
$$

Correspondingly, the optical theorem gives, for $a \neq b$,

$$
\begin{aligned}
\sigma_{\mathrm{tot}}(s) & =\frac{4 \pi}{k} \operatorname{Im}(4 F(s, 0) / \sqrt{s}) \\
& =\frac{4 \pi}{k^{2}} \sum_{l=0}^{\infty}(2 l+1) \operatorname{Im} a_{l}(s) .
\end{aligned}
$$

For identical particles $a=b$, e.g., for $\pi^{0} \pi^{0}$ scattering, or for pion-pion scattering with isospin $I$, we have the same formula for the differential cross section,

$$
\frac{d \sigma}{d \Omega}(s, t)=\left|4 \frac{F(s, t)}{\sqrt{s}}\right|^{2},
$$

and the same form of the unitarity constraint,

$$
\operatorname{Im} a_{l}^{I}(s) \geq\left|a_{l}^{I}(s)\right|^{2}, \quad s \geq 4,
$$

but the partial waves $a_{l}(s) \rightarrow 2 a_{l}^{I}(s)$ in the partial wave expansion, i.e.,

$$
F^{I}(s, t)=\frac{\sqrt{s}}{4 k} \sum_{l=0}^{\infty}(2 l+1) 2 a_{l}^{I}(s) P_{l}(z) .
$$

With this normalization, $F^{I}(4,0)=a_{0}^{I}$, the $S$-wave scattering length for isospin $I$, and for pion-pion scattering the identical particle factors lead to

$$
\sigma_{\mathrm{tot}}^{I}(s)=\frac{4 \pi}{k^{2}} \sum_{l=0}^{\infty}(2 l+1) 2 \operatorname{Im} a_{l}^{I}(s) .
$$

In the following, we shall consider nonidentical particles $a \neq b$ for detailed derivations and quote the identical particle results when needed.

\section{CONVEXITY PROPERTIES OF LOWER BOUND ON ABSORPTIVE PART IN TERMS OF TOTAL CROSS SECTION}

Martin has proved unitarity lower bounds on $A(s, t)$ for $0<t<t_{0}$ in terms of $\sigma_{\text {tot }}(s)$ [2], and in terms of $\sigma_{\text {inel }}(s)$ [16]. He has also proved [20] that these bounds are convex functions of $\sigma_{\text {tot }}(s)$ and $\sigma_{\text {inel }}(s)$, respectively. We recall first the convexity properties which will be crucial for our proofs of lower bounds on $C(t)$ in terms of energy averages of total cross sections. We work at a fixed $s$, and suppress the $s$ dependence of $\operatorname{Im} a_{l}(s)$, and $\sigma_{\text {tot }}(s)$ for simplicity of writing. Using $0 \leq \operatorname{Im} a_{l} \leq 1$, the lower bound on $A(s, t)$ for given $\sigma_{\text {tot }}$ is obtained by choosing

$$
\begin{aligned}
& \operatorname{Im} a_{l}=1, \quad 0 \leq l \leq L ; \quad \operatorname{Im} a_{L+1}=\eta ; \\
& \operatorname{Im} a_{l}=0, \quad l>L+1,
\end{aligned}
$$

where the fraction $\eta(0 \leq \eta<1)$ and the integer $L$ are determined from the given $\sigma_{\text {tot }}$. Thus,

$$
\begin{aligned}
A(s, t) \frac{4 k}{\sqrt{s}} & \geq\left(\sum_{l=0}^{L}(2 l+1) P_{l}(z)+\eta(2 L+3) P_{L+1}(z)\right) \\
& \equiv A(z)
\end{aligned}
$$

where 


$$
\sigma_{\text {tot }} \frac{k^{2}}{4 \pi}=\left(\sum_{l=0}^{l=L}(2 l+1)+\eta(2 L+3)\right) \equiv \Sigma_{\text {tot }} .
$$

Hence, $A(z)$ is a monotonically increasing function of $\Sigma_{\text {tot }}$ with piecewise constant positive derivative. Denoting $\operatorname{Int}(x)=$ integer part of $x$,

$$
d A(z) / d \Sigma_{\mathrm{tot}}=P_{L+1}(z), \quad L=\operatorname{Int}\left(\sqrt{\Sigma_{\mathrm{tot}}}\right)-1,
$$

which increases with $L$ since $z>1$, and hence with $\Sigma_{\text {tot }}$ when it crosses the square of an integer. This proves that the lower bound $A(z)$ is a convex function of $\Sigma_{\text {tot }}$, and that

$$
A(z)=\Sigma_{\text {tot }}, \quad \text { for } \Sigma_{\text {tot }} \leq 1,
$$

and, for $\Sigma_{\text {tot }}>1$,

$$
\begin{aligned}
A(z) & =1+\int_{1}^{\Sigma_{\mathrm{tot}}} P_{\mathrm{Int}(\sqrt{\sigma})}(z) d \sigma, \\
& \geq 1+2 \int_{0}^{\sqrt{\Sigma_{\mathrm{tot}}}-1}(\mu+1) P_{\mu}(z) d \mu .
\end{aligned}
$$

Using integral representations for $P_{\mu}(z)$ and for the modified Bessel function $I_{0}$, we obtain for $\mu \geq 0, z>1$,

$$
P_{\mu}(z) \geq I_{0}\left(\mu \ln z_{+}\right), \quad z_{+} \equiv z+\sqrt{z^{2}-1} .
$$

This yields the strict inequality (without any high energy approximation),

$$
\begin{aligned}
A(z) \geq & \left.2\left(\frac{x I_{1}(x)}{\left(\ln z_{+}\right)^{2}}+\frac{I_{0}(x)}{\ln z_{+}}\right)\right|_{x=\left(\sqrt{\Sigma_{\mathrm{tot}}}-1\right) \ln z_{+}} \\
& +1+2 \ln z_{+}, \quad \text { for } \Sigma_{\mathrm{tot}}>1 .
\end{aligned}
$$

At high energy, this gives

$$
A(s, t)>\left.\frac{s}{4 t} x I_{1}(x)\right|_{x=\sqrt{t \tau_{\mathrm{tot}}(s) /(4 \pi)}}(1+O(1 / \sqrt{s})),
$$

which is a convex function of $\sigma_{\mathrm{tot}}(s)$.

\section{UPPER BOUND ON ENERGY-AVERAGED TOTAL CROSS SECTION}

Defining

$$
\bar{\sigma}_{\text {tot }}(s, \infty) \equiv s \int_{s}^{\infty} \frac{d s^{\prime}}{s^{\prime 2}} \sigma_{\text {tot }}\left(s^{\prime}\right)
$$

and

$$
C_{s}(t) \equiv \int_{s}^{\infty} d s^{\prime} A\left(s^{\prime}, t\right) / s^{3}<\infty, \quad 0 \leq t<t_{0},
$$

we obtain

$$
\begin{aligned}
C_{s}(t) & \geq \frac{1}{4 t s} s \int_{s}^{\infty} \frac{d s^{\prime}}{s^{\prime 2}} \sqrt{\frac{t \sigma_{\mathrm{tot}}\left(s^{\prime}\right)}{4 \pi}} I_{1}\left(\sqrt{\frac{t \sigma_{\mathrm{tot}}\left(s^{\prime}\right)}{4 \pi}}\right) \\
& \geq \frac{1}{4 t s} \sqrt{\frac{t \bar{\sigma}_{\mathrm{tot}}(s, \infty)}{4 \pi}} I_{1}\left(\sqrt{\frac{t \bar{\sigma}_{\mathrm{tot}}(s, \infty)}{4 \pi}}\right),
\end{aligned}
$$

since the average of a convex function must be greater than the convex function of the average [21]. At high energies if $\bar{\sigma}_{\text {tot }}(s, \infty)$ goes to $\infty$, the asymptotic expansion of $I_{1}(\xi)$ yields

$$
4 s t C_{s}(t) \sqrt{2 \pi}>(\sqrt{\xi} \exp \xi)(1+O(1 / \xi)),
$$

where

$$
\xi \equiv \sqrt{\frac{t \bar{\sigma}_{\mathrm{tot}}(s, \infty)}{4 \pi}} .
$$

To extract a bound on the cross section, we need the following lemma [20]. If $\xi>1$, and

$$
y \geq \sqrt{\xi} \exp \xi,
$$

then

$$
\xi<f(y) \equiv \ln y-(1 / 2) \ln \left(\ln y-\frac{1}{2} \ln \ln y\right) .
$$

Proof. It is enough to prove this for $y=\sqrt{\xi} \exp \xi$, since the right-hand side is an increasing function of $\xi$. Taking logarithms, and using $\xi=\ln y-(1 / 2) \ln \xi \equiv \xi_{1}$ repeatedly,

$$
\xi=\ln y-(1 / 2) \ln \left(\ln y-\frac{1}{2} \ln \xi_{1}\right) .
$$

For fixed $y$, the derivative of the right-hand side with respect to $\xi_{1}$ is $\left(4 \xi_{1}^{2}\right)^{-1}$, which is positive, and $\xi_{1}<\ln y$ for $\xi>1$. Hence, the stated upper bound on $\xi$ follows.

Instead of the $s$-dependent $C_{s}(t)$, we shall use the simple $s$-independent upper bound,

$$
C_{s}(t) \leq C(t)-\int_{4}^{x} d s^{\prime} \frac{k^{\prime} \sqrt{s^{\prime}} \sigma_{\mathrm{tot}}\left(s^{\prime}\right)}{\left(s^{\prime}\right)^{3} 16 \pi}, \quad 4<x<s,
$$

which follows by using $A(s, t)>A(s, 0)$ for $4>t>0$ and improves the value $C(t)$ if low energy total cross sections are known. The integral of the weight function multiplying $\sigma_{\text {tot }}$ can be done. Thus,

$$
C_{s}(t) \leq C_{x}(t) \equiv C(t)-\frac{(x-4)^{3 / 2} \bar{\sigma}_{\text {tot }}(x)}{12 x^{3 / 2} 16 \pi},
$$

where 


$$
\bar{\sigma}_{\text {tot }}(x)=\frac{\int_{4}^{x} d s^{\prime} k^{\prime} \sqrt{s^{\prime}} \sigma_{\mathrm{tot}}\left(s^{\prime}\right) / s^{\prime 3}}{\int_{4}^{x} d s^{\prime} k^{\prime} \sqrt{s^{\prime}} / s^{\prime 3}} .
$$

With $f(y)$ as defined above, the upper bound on the average total cross section in terms of $C_{x}(t)$ is

$$
\begin{gathered}
\bar{\sigma}_{\text {tot }}(s, \infty) \leq_{s \rightarrow \infty} \frac{4 \pi}{t}\left(f\left(s / s_{0}\right)+O\left(\ln \left(s / s_{0}\right)\right)^{-1}\right)^{2}, \\
\frac{1}{s_{0}}=4 t C_{x}(t) \sqrt{2 \pi}, \quad t=4 m_{\pi}^{2}-\epsilon .
\end{gathered}
$$

We may also find bounds on the average of the total cross section in the interval $(s, 2 s)$,

$$
\bar{\sigma}_{\mathrm{tot}}(s, 2 s) \equiv 2 s \int_{s}^{2 s} \frac{d s^{\prime}}{s^{\prime 2}} \sigma_{\mathrm{tot}}\left(s^{\prime}\right)
$$

The lower bound on $A(s, t)$ and its convexity yield

$$
\begin{aligned}
C_{x}(t) & \geq \frac{1}{8 t s} 2 s \int_{s}^{\infty} \frac{d s^{\prime}}{s^{\prime 2}} \sqrt{\frac{t \sigma_{\mathrm{tot}}\left(s^{\prime}\right)}{4 \pi}} I_{1}\left(\sqrt{\frac{t \sigma_{\mathrm{tot}}\left(s^{\prime}\right)}{4 \pi}}\right) \\
& \geq \frac{1}{8 t s} \sqrt{\frac{t \bar{\sigma}_{\mathrm{tot}}(s, 2 s)}{4 \pi}} I_{1}\left(\sqrt{\frac{t \bar{\sigma}_{\mathrm{tot}}(s, 2 s)}{4 \pi}}\right) .
\end{aligned}
$$

Asymptotically we obtain a bound of the same form as before, but with the scale factor in the logarithm being $s_{0} / 2$,

$$
\bar{\sigma}_{\text {tot }}(s, 2 s) \leq_{s \rightarrow \infty} \frac{4 \pi}{t}\left(f\left(2 s / s_{0}\right)+O\left(\ln \left(s / s_{0}\right)\right)^{-1}\right)^{2} .
$$

Note that $\sigma_{\text {tot }}(s)<\bar{\sigma}_{\text {tot }}(s, 2 s)$ if the cross section increases with $s$ in the interval $(s, 2 s)$; the above bound on energy averages therefore immediately yields a bound on $\sigma_{\mathrm{tot}}(s)$ in that case.

For identical particles there are only even partial waves in the partial wave expansions, but the lower bound on the absorptive part is again a convex function of the total cross section; the identical particle factors multiplying the partial waves ensure that in spite of only even partial waves contributing, the largest partial wave $L$ in the variational bound which is of $O\left(\sqrt{s \sigma_{\text {tot }}(s)}\right)$ has only $O(1)$ corrections with respect to the nonidentical particles case. The quoted asymptotic bounds on the absorptive part in terms of $\sigma_{\text {tot }}$ and on the energy-averaged total cross section in terms of $C_{x}(t)$ therefore remain unchanged.

\section{PHENOMENOLOGICAL BOUNDS IN TERMS OF $D$-WAVE SCATTERING LENGTH}

Rigorous results from axiomatic field theory do not guarantee finiteness of the $D$-wave scattering lengths. However if we use phenomenological values for them we can choose $\epsilon=0$ and evaluate $C(t=4)$. We shall use $F^{\pi^{+} \pi^{0} \rightarrow \pi^{+} \pi^{0}}=1 / 2\left(F^{1}+F^{2}\right), F^{\pi^{0} \pi^{0} \rightarrow \pi^{0} \pi^{0}}=\frac{1}{3} F^{0}+\frac{2}{3} F^{2}$, the crossing relation,

$$
\frac{1}{2}\left(F^{1}+F^{2}\right)(s, t)=\frac{1}{3}\left(F^{0}-F^{2}\right)(t, s),
$$

and the total crossing symmetry of the $\pi^{0} \pi^{0} \rightarrow \pi^{0} \pi^{0}$ amplitude. If we denote $F(s, t)=G(t, s)=G\left(t ; z_{t}\right)$, where $F(s, t)$ denotes the $\pi^{+} \pi^{0} \rightarrow \pi^{+} \pi^{0}$ or the $\pi^{0} \pi^{0} \rightarrow \pi^{0} \pi^{0}$ amplitude, then the corresponding $G(t, s)$ has only even partial waves,

$$
g_{l}(t)=\frac{1}{2} \int_{-1}^{1} d z_{t} P_{l}\left(z_{t}\right) G\left(t ; z_{t}\right) .
$$

For $0<t<4$, with the absorptive part $F_{s}(s, t)$ defined by Eq. (7), the fixed- $t$ dispersion relations with two subtractions imply the Froissart-Gribov formula rigorously for $l \geq 2$,

$$
g_{l}(t)=\frac{4}{\pi(4-t)} \int_{4}^{\infty} d s^{\prime} Q_{l}\left(\frac{2\left(s^{\prime}-4\right)+4+t}{4-t}\right) F_{s}\left(s^{\prime}, t\right),
$$

where $Q_{l}$ denotes the Legendre function of the second kind. The positivity of the absorptive part then implies the positivity of $g_{l}(t)$ for $0<t<4$; further,

$$
g_{2}(t) \rightarrow_{t \rightarrow 4} \frac{(t-4)^{2}}{15 \pi} \int_{4}^{\infty} d s^{\prime} \frac{F_{s}\left(s^{\prime}, t\right)}{s^{\prime 3}} .
$$

If the $t$-channel $D$-wave scattering lengths exist, the definitions of $C(t)$ and of the $D$-wave scattering lengths $a_{2}^{I}$ for isospin $I$ yield

$$
C^{\pi^{+} \pi^{0} \rightarrow \pi^{+} \pi^{0}}(t=4)=\frac{5 \pi}{16} m_{\pi}\left(a_{2}^{0}-a_{2}^{2}\right),
$$

and

$$
C^{\pi^{0} \pi^{0} \rightarrow \pi^{0} \pi^{0}}(t=4)=\frac{5 \pi}{16} m_{\pi}\left(a_{2}^{0}+2 a_{2}^{2}\right) .
$$

Here we have defined the $l$-wave scattering lengths $a_{l}^{I}$ as the $q \rightarrow 0$ limits of the phase shifts $\delta_{l}^{I}(q)$ divided by $q^{2 l+1}$ where $q$ is the c.m. momentum. Then an $S$-wave scattering length is indeed a length, with dimension $m_{\pi}^{-1}$, and the $D$-wave scattering lengths have dimension $m_{\pi}^{-5}$. Then, phenomenologically [19] we have

$$
a_{2}^{0} \approx 0.00175 m_{\pi}^{-5} ; \quad a_{2}^{2} \approx 0.00017 m_{\pi}^{-5},
$$

and Roy [14] has obtained from low energy data, for $x=50$,

$$
\bar{\sigma}_{\mathrm{tot}}^{\pi^{0} \pi^{0}}(x)=8.2 \pm 4 m b ; \quad \bar{\sigma}_{\mathrm{tot}}^{\pi^{+}} \pi^{0}(x)=17 \pm 3.5 \mathrm{mb} .
$$

With $\epsilon=0, t=4$, and the values of $C_{x}(t=4)$ given in terms of the scattering lengths, and the low energy total cross sections, we have, from Eqs. (31)-(33), with $x=50$, 


$$
\begin{aligned}
\pi^{0} \pi^{0}: & s_{0}=17 m_{\pi}^{2}, \\
C_{x}(4) & =2.05 \times 10^{-3}-0.6 \times 10^{-3} \\
& =1.45 \times 10^{-3} m_{\pi}^{-4}, \\
\pi^{+} \pi^{0}: & s_{0}=81 m_{\pi}^{2}, \\
C_{x}(4) & =1.55 \times 10^{-3}-1.24 \times 10^{-3} \\
& =.31 \times 10^{-3} m_{\pi}^{-4},
\end{aligned}
$$

where we have indicated the separate contributions of the $D$-wave scattering lengths and low energy total cross sections to $C_{x}(4)$ but have not indicated the (substantial) errors on them which imply corresponding errors on the scale factors. Our bounds on average total cross sections for $\pi^{+} \pi^{0}$ and $\pi^{0} \pi^{0}$ scattering therefore do not contain any unknown constants, but the scale factor $s_{0}$ has large phenomenological errors. We cure this problem in the next section at the cost of getting poorer bounds.

\section{ABSOLUTE BOUNDS ON THE $D$-WAVE BELOW THRESHOLD FOR $\pi^{0} \pi^{0}$ SCATTERING}

Although threshold behavior cannot be proved from first principles, it was shown long ago [22] that $\left|f_{l}(s)\right|<$ $C(4-s)^{l-1}$ must hold for $0<s<4$. We derive an absolute bound of this form and use it to derive a rigorous asymptotic bound on energy-averaged total cross section for $\pi^{0} \pi^{0}$ scattering without unknown constants. As noted already, for $0<s<4$ and $l \geq 2$, the Froissart-Gribov formula implies that $f_{l}(s)>0$. Hence, for $0<s<4$, $4-s<t<4$, the convergent partial wave expansion,

$$
\begin{aligned}
F(s, t)-F(s, 0)= & \Sigma_{l=2}^{\infty}(2 l+1) f_{l}(s) \\
& \times\left(P_{l}\left(\frac{2 t-4+s}{4-s}\right)-1\right),
\end{aligned}
$$

is in fact a sum of positive terms and yields an upper bound for the $l \geq 2$ partial waves if we can obtain a bound on $F(s, t)-F(s, 0)$ using analyticity. The twice subtracted fixed- $t$ dispersion relations in $s$ can be rewritten in terms of the convenient variable $z \equiv(s-2+t / 2)^{2}$, with $F(s, t) \equiv F(z ; t)$. For $0 \leq t<4$,

$$
F(s, t)-F\left(\frac{4-t}{2}, t\right)=\frac{z}{\pi} \int_{z_{0}}^{\infty} d z^{\prime} \frac{\operatorname{Im} F\left(z^{\prime} ; t\right)}{z^{\prime}\left(z^{\prime}-z\right)},
$$

and the positivity of the absorptive part then yields

$$
F(s, t)-F\left(\frac{4-t}{2}, t\right) \geq 0, \quad \text { if } 0 \leq z<z_{0}=\left(2+\frac{t}{2}\right)^{2}
$$

If $s_{1}<s<4$ and $z_{1} \equiv\left(s_{1}-2+t / 2\right)^{2}$, then

$$
\begin{aligned}
z_{1}-z & =\left(s_{1}-s\right)\left(s_{1}+s-4+t\right)<0, \\
& \text { if } t>4-s-s_{1},
\end{aligned}
$$

and hence for $z_{1}<z<z_{0}$,

$$
\begin{aligned}
& \left(z^{\prime}-z\right)^{-1}-\left(z_{0}-z_{1}\right)\left(\left(z_{0}-z\right)\left(z^{\prime}-z_{1}\right)\right)^{-1} \\
& \quad=\left(z^{\prime}-z_{0}\right)\left(z_{1}-z\right)\left(\left(z^{\prime}-z\right)\left(z_{0}-z\right)\left(z^{\prime}-z_{1}\right)\right)^{-1}<0 .
\end{aligned}
$$

Inserting this into the dispersion relation we have, for $4>t>4-s-s_{1}$, and $t \geq 0$,

$$
\begin{aligned}
F(s, t)- & F\left(\frac{4-t}{2}, t\right)<\frac{\left(4-s_{1}\right)\left(s_{1}+t\right)^{2}\left(s-2+\frac{t}{2}\right)^{2}}{(4-s)(s+t)^{2}\left(s_{1}-2+\frac{t}{2}\right)^{2}} \\
& \times\left(F\left(s_{1}, t\right)-F\left(\frac{4-t}{2}, t\right)\right), \quad \text { if } s_{1}<s<4 .
\end{aligned}
$$

Choosing $s_{1}=3, t=2$, and $3<s<4$, we get

$$
F(s, 2)-F(1,2)<(25 / 16)(F(3,2)-F(1,2)) /(4-s) \text {. }
$$

Using this and $F(s, 0)>F(2,0)$,

$$
\begin{aligned}
& F(s, 2)-F(s, 0)<F(1,2)-F(2,0) \\
& \quad+(25 / 16)(F(3,2)-F(1,2)) /(4-s), \text { for } 3<s<4 .
\end{aligned}
$$

We now use absolute bounds on pion-pion amplitudes first discovered by Martin [23], and improved successively by [4], [24], and [25] in the improved final form,

$$
\begin{aligned}
-7.25 & <F(1,2)<2.75, \\
F(2,0) & >-3.5, \quad F(3,2)<14.5,
\end{aligned}
$$

with normalization $F(4,0)=$ S-wave scattering length, and we obtain the absolute bound,

$$
F(s, 2)-F(s, 0)<6.25+\frac{33.99}{4-s}, \text { for } 3<s<4 .
$$

The partial wave expansion of $F(s, 2)-F(s, 0)$ now yields, for $3<s<4$,

$$
f_{l}(s) \leq \frac{6.25+\frac{33.99}{4-s}}{(2 l+1)\left(P_{l}\left(\frac{s}{4-s}\right)-1\right)},
$$

which implies in particular,

$$
f_{2}(s)<_{s \rightarrow 4-} \frac{4-s}{120}\left(34+6.25(4-s)+O(4-s)^{2}\right) .
$$


With $s$ replaced by $t$ in this formula, the Froissart-Gribov formula now yields

$$
C^{\pi^{0} \pi^{0} \rightarrow \pi^{0} \pi^{0}}(t)<_{t \rightarrow 4-} \frac{17 \pi}{4(4-t)} .
$$

\section{ABSOLUTE BOUND ON ENERGY-AVERAGED TOTAL CROSS SECTION FOR $\pi^{0} \pi^{0}$ SCATTERING AT HIGH ENERGY}

Inserting the bound on $C(t)$ into the average crosssection bound, the optimum value of $t$ turns out to be $t=4-\left(1 / 8 \ln \left(s / s_{0}\right)\right)^{-1}$, and the optimum bound,

$$
\begin{aligned}
\bar{\sigma}_{\text {tot }}(s, \infty) & \leq \pi\left(m_{\pi}\right)^{-2}\left[\ln \left(s / s_{0}\right)+(1 / 2) \ln \ln \left(s / s_{0}\right)+1\right]^{2} \\
& +O\left(\ln \ln \left(s / s_{0}\right)\right), \quad s_{0}^{-1}=17 \pi \sqrt{\pi / 2} m_{\pi}^{-2} .
\end{aligned}
$$

For $\bar{\sigma}_{\text {tot }}(s, 2 s)$ we obtain the same form of the bound, but with half the value of $s_{0}$.

\section{OUTLOOK}

Our basic bound on the absorptive part, Eq. (20), is valid at all energies and its energy integral may be used for comparisons with experimental cross-section data which have a large nonasymptotic contribution at current energies. We have highlighted the simpler asymptotic upper bounds on average total cross sections.

We believe that our result is important as a matter of principle. However, we also believe that the magnitude of the coefficient in front of the Froissart bound is not satisfactory, especially if one decides to believe that the
Froissart term is universal and compares with p-p and $p-\bar{p}$ cross sections at the ISR [26], the SppbarS [27], the Tevatron [28] and the LHC [29]. All these indicate the existence of a Froissart-like contribution with a much smaller coefficient and a much larger scale, and they are well reproduced by, for instance, the Bourrely-Soffer-Wu model [15] which incorporates automatically the Froissart behavior. Returning to $\pi \pi$ scattering, can the situation be improved? Yes, because one has to enforce crossing symmetry and unitarity. Kupsch [30] has constructed a crossing symmetric model satisfying Eq. (8) but never tried to get numerical results. Also, we believe that unitarity in the elastic strips could be important. This led to the discovery by Gribov [31] that the behavior $s F(t)$ for the total amplitude is impossible. If you remove the elastic unitarity constraint [32], the Gribov theorem disappears. To attack the problem, one could use a variational approach, taking as an input the inelastic double spectral function in the Mandelstam representation. All we need is to find someone courageous not looking for a job.

Similar bounds on inelastic cross sections without any unknown constants will be reported separately [33].

\section{ACKNOWLEDGEMENTS}

A. M. would like to thank the members of ITEP (Moscow) for inviting him to give a talk in 2010 which resurrected his interest in the subject, and also Tai Tsun $\mathrm{Wu}$ and Raymond Stora for stimulating discussions. S. M. R. wishes to thank the Indian National Science Academy for the INSA Senior Scientist award and L. Alvarez-Gaume for an invitation to CERN in 2009 which helped start this collaboration.
[1] M. Froissart, Phys. Rev. 123, 1053 (1961).

[2] A. Martin, Nuovo Cimento A 42, 930 (1966).

[3] H. Lehmann, Nuovo Cimento 10, 579 (1958); Fortschr. Phys. 6, 159 (1959).

[4] L. Lukaszuk and A. Martin, Nuovo Cimento A 52, 122 (1967).

[5] Y. S. Jin and A. Martin, Phys. Rev. 135, B1375 (1964).

[6] J. D. Bessis and V. Glaser, Nuovo Cimento A 50, 568 (1967); G. Sommer, Nuovo Cimento A 48, 92 (1967); for reviews, see G. Sommer, Fortschr. Phys. 18, 577 (1970); A. Martin, Report No. CERN-TH/99-110, 1999.

[7] See, e.g., R. F. Streater and A. S. Wightman, PCT. Spin and Statistics and All That (Princeton University Press, Princeton, NJ, 2000).

[8] H. Epstein, V. Glaser, and A. Martin, Commun. Math. Phys. 13, 257 (1969).

[9] R. Haag and D. Kastler, J. Math. Phys. 5, 848 (1964); D. Ruelle, Helv. Phys. Acta 35, 147 (1962); H. Araki,
Mathematical Theory of Quantum Fields (Oxford University Press, Oxford, 2000).

[10] Y. I. Azimov, Phys. Rev. D 84, 056012 (2011).

[11] A. Martin, in Proceedings of the 1962 conference on high energy physics at CERN, edited by J. Prentki (CERN, GENEVA, 1962), pp. 566-567; A. Martin, Phys. Rev. 129, 1432 (1963).

[12] W. Zimmermann, Nuovo Cimento 10, 597 (1958); 21, 249 (1961); 21, 268 (1961).

[13] V. Singh and S. M. Roy, Ann. Phys. (N.Y.) 57, 461 (1970).

[14] S. M. Roy, Phys. Rep. 5, 125 (1972).

[15] H. Cheng and T. T. Wu, Phys. Rev. Lett. 24, 1456 (1970); C. Bourrely, J. Soffer, and T. T. Wu, Phys. Rev. D 19, 3249 (1979); Nucl. Phys. B247, 15 (1984); M. M. Block, Phys. Rep. 436, 71 (2006), and references therein.

[16] A. Martin, Phys. Rev. D 80, 065013 (2009). 
[17] T. T. Wu, A. Martin, S. M. Roy, and V. Singh, Phys. Rev. D 84, 025012 (2011).

[18] F. J. Yndurain, Phys. Lett. 31B, 368 (1970); A. K. Common, Nuovo Cimento A 69, 115 (1970).

[19] G. Colangelo, J. Gasser, and H. Leutwyler, Nucl. Phys. B603, 125 (2001); B. Ananthanarayan, G. Colangelo, J. Gasser, and H. Leutwyler, Phys. Rep. 353, 207 (2001).

[20] A. Martin, Pomeranchuk prize lecture (ITEP, Moscow, 2010); https://cds.cern.ch/record/1591290.

[21] G. H. Hardy, J. E. Littlewood, and G. Pólya, Inequalities (Cambridge University Press, Cambridge, England, 1952), p. 74 .

[22] A. Martin, Nuovo Cimento A 47, 265 (1967).

[23] A. Martin, High Energy Physics and Elementary Particles (IAEA, Vienna, 1965), p. 155.

[24] G. Auberson, L. Epele, G. Mahoux, and F. R. A. Simao, Nucl. Phys. B73, 314 (1974); B94, 311 (1975).

[25] C. Lopez and G. Mennessier, Phys. Lett. 58B, 437 (1975); Nucl. Phys. B118, 426 (1977).
[26] U. Amaldi, R. Biancastelli, C. Bosio, G. Matthiae, J. V. Allaby, W. Bartel, G. Cocconi, A.N. Diddens, R. W. Dobinson, and A. M. Wetherell, Phys. Lett. 44B, 112 (1973); S. R. Amendolia et al., Phys. Lett. 44B, 119 (1973).

[27] M. Bozzo et al., Phys. Lett. B 147, 392 (1984); C. Augier et al., Phys. Lett. B 316, 448 (1993).

[28] N. A. Amos et al., Phys. Rev. Lett. 63, 2784 (1989); Phys. Lett. B 243, 158 (1990); Phys. Rev. Lett. 68, 2433 (1992).

[29] Totem Collaboration, Europhys. Lett. 101, 21004 (2013); Atlas-Alpha Collaboration (to be published).

[30] J. Kupsch, Nuovo Cimento A 71, 85 (1982).

[31] V. N. Gribov, in Proc. International Conf. on High Energy Physics at Rochester, edited by E. C. G. Sudershan, J. H. Tinlot, and A. C. Melissos (University of Rochester, Rochester, NY, 1960), p. 340.

[32] A. Martin and J.-M. Richard, in Proceedings of Forward Physics and Luminosity Determination at LHC, Helsinki, 2000, edited by K. Haiti et al. (World Scientific, Singapore, 2001), p. 27.p

[33] A. Martin and S. M. Roy (to be published). 